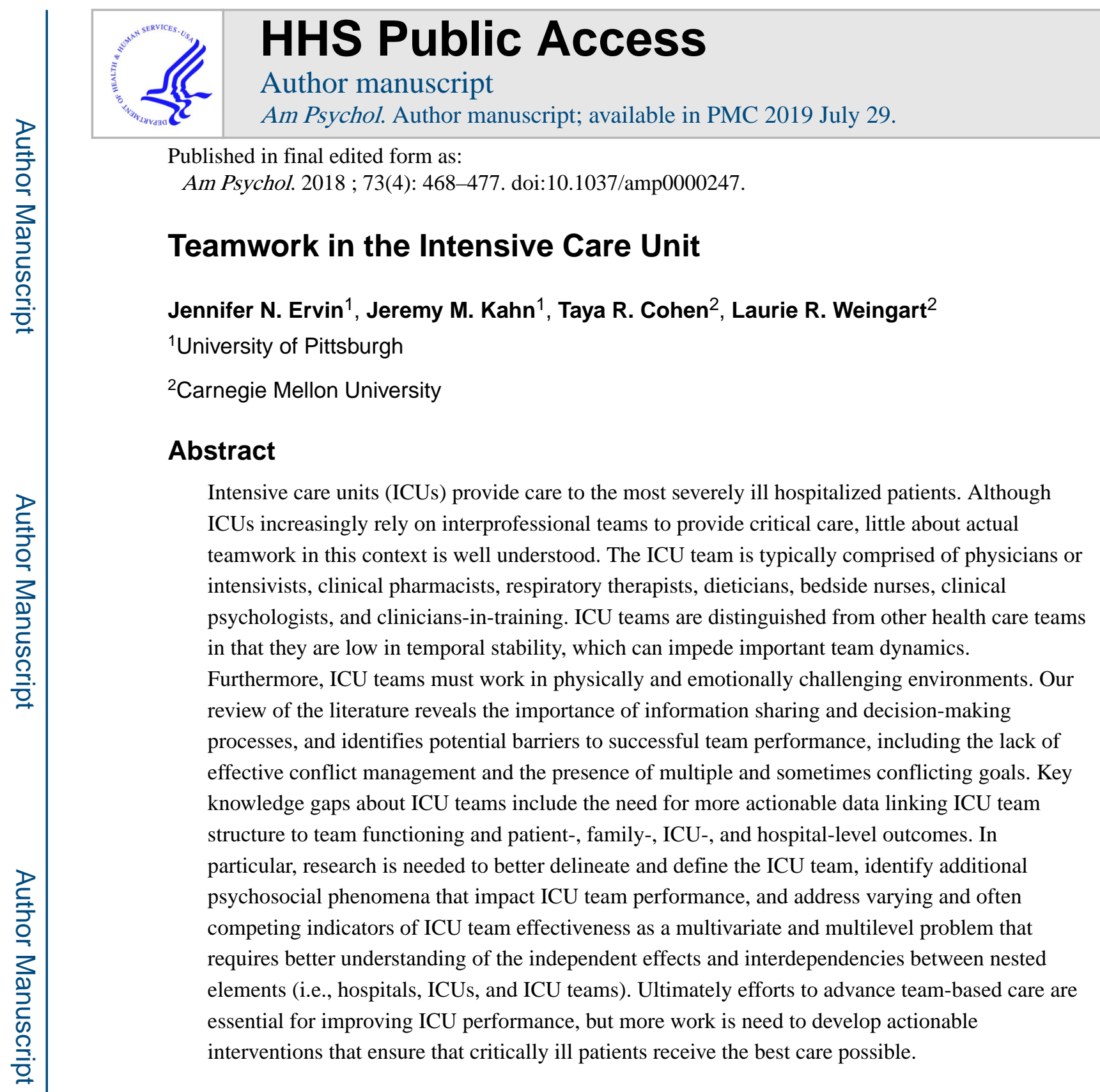

\title{
Keywords
}

Teamwork; performance; critical care; patient care; collaboration

\section{Teamwork in the Intensive Care Unit}

The intensive care unit (ICU) is a specialized hospital unit dedicated to the care of patients requiring life-support and those at extremely high risk for organ failure and death.

Approximately 5.7 million individuals are admitted to an ICU in the United States each year (Barrett, Smith, Elixhauser, Honigman, \& Pines, 2011). ICUs care for the most severely ill hospitalized patients, and in doing so are one of the most resource demanding and stressful areas of the hospital. The field of critical care medicine has embraced a standard whereby care is provided by an interprofessional team of clinicians (Weled et al., 2015). Under such a model, intensivists (i.e., physicians with specialized training in intensive care medicine) or other types of attending physicians collaborate with and capitalize on the interprofessional 
expertise of bedside nurses, respiratory therapists, clinical pharmacists, dieticians, and clinical psychologists (Durbin, 2006).

Recent shifts toward interprofessional care have resulted in lower morbidity and mortality rates among ICU patients (Curtis et al., 2006). However, little about actual teamwork in the ICU is well understood. Few existing studies focus on the form and function of interprofessional collaborations among critical care clinicians or provide insight as to how these relationships influence team performance. As a result, critical care providers lack guidance on how to leverage team function in order to improve patient outcomes and reduce health care costs in the ICU. In this review we address this gap by synthesizing extant research on teamwork in the ICU and providing a roadmap for future research in this domain. First, we describe the extent to which ICU teams are similar to and yet distinct from teams in other organizational contexts. Second, we review the existing research on team performance in the ICU. Third, we highlight key areas for future study, including outcome indicators that reflect effective team processes.

\section{Characteristics of Intensive Care Teams}

The social scientific definition of a team is "a distinguishable set of 2 or more people who interact dynamically, interdependently, and adaptively toward a common and valued goal/ object/mission, who have each been assigned specific roles or functions to perform" (Salas, Dickson, Converse, \& Tannenbaum, 1992, p. 4). When defined in this manner, effective team performance is contingent upon conflict, cooperation, coordination, coaching, communication, and cognition between team members (Salas, Shuffler, Thayer, Bedwell, \& Lazzara, 2015). Analogously, team-based medicine refers to care that requires the expertise and coordinated efforts of two or more clinicians. Successful ICU teams are able to exchange information and work together on some shared goal or task, such as managing an artificial respirator, providing intravenous sedation to keep patients comfortable, or dealing with the emotional distress of family members whose loved ones are critically ill.

There is no single defining feature that makes the ICU a unique context for collective behavior; rather, ICU clinicians face a combination of specific structural and situational demands that differentiates them from members of other organizational and healthcare teams. These include but are not limited to differences in the lifespan of ICU teams and the physical and emotional challenges faced by those working in the ICU (e.g., Salas et al., 2015). With regard to lifespan, ICU teams are low in temporal stability in that the identity of individual team members changes from day to day (Alexanian, Kitto, Rak, \& Reeves, 2015; Andreatta, 2010; Hughes et al., 2016). Yet unlike other health care teams that are low in temporal stability, such as cardiac resuscitation teams or trauma teams, the tasks for ICU teams are longer in duration than the life of each team. For example, a single patient might spend 14 days or longer in an ICU, during which time team composition and related dynamics are in flux (Wildman, Shuffler, Lazzara, Fiore, \& Burke, 2012).

ICU teams function in spite of low temporal stability because the persons fulfilling each position are expected to bring shared knowledge about caring for critically ill patients and shared expectations about their specific roles in the ICU (Alexanian et al., 2015). Formal and informal hand-off processes also contribute to team functioning in this setting of variable 
team membership. Formally, hand-off protocols can improve the efficiency and effectiveness of face-to-face communication during shift changes, reducing errors, improving continuity and reducing uncertainty about patient goals of care (Patterson, Roth, Woods, Chow, \& Gomes, 2004). Informally, unstructured handoffs (Ong, BiomedE, \& Coicera, 2011) and electronic health records that, if properly maintained, allow clinicians to access, update, and manage patient information, contribute to continuity of care (Hoover, 2017).

ICU teams are also distinguished by the unique physical and emotional constraints that affect team performance. With regard to the physical environment of the ICU, near constant alarms, uneven lighting, poorly placed equipment, and space limitations mean that the physical environment is at best not helpful and at worst harmful to the goals of team-based critical care (Alameddine et al., 2008; Salas, Cooke, \& Rosen, 2008; Shortell et al., 1994; Xie \& Carayon, 2015). Perhaps in response to the substantial variation that exists in the use of physical space, as well as the recognition that many hospitals and ICUs do not use space optimally, hospital and ICU design is a burgeoning area of multidisciplinary research (e.g., Rashid, 2006; Thompson et al., 2012). Early results from this research suggest that open floor plans, easy circulation around the patient's bed, clear lines of sight between nursing stations and rooms, and single-patient rooms can improve communication and family satisfaction with care (Kesecioglu, Schneider, van der Kooi, \& Bion, 2012).

With regard to the emotional constraints, death and dying is a daily occurrence in the ICU, forcing the team to function in a highly charged emotional environment characterized by persistent grieving and moral distress (Embriaco, Papazian, Kentish-Barnes, Pochard, \& Azoulay, 2007; Henrich et al., 2016). Despite having access to support, emotional distress is common among care providers and spreads easily to other team members, which can increase collective anxiety and reduce team performance (Picquette, Reeves, \& LeBlanc, 2009).

These unique characteristics of ICU teams have several implications for critical care. First, effective communication is a necessity. In this context, effective communication refers to the ability to transfer information, ideas, and opinions across team members with widely varying backgrounds, experiences, and skill-levels (e.g., Reader, Flin, Mearns, \& Cuthbertson, 2009). Second, ICU team members must trust in others' knowledge, skills, and training so that teams can perform in high stakes situations despite little or no shared history (Hughes et al., 2016; Wildman, et al., 2012). Third, team leadership should balance authority with inclusiveness while establishing shared goals and fostering sense of shared responsibility for patient care (Fernandez \& Grand, 2015; Manthous \& Hollingshead; 2011; Manthous, Nembhard, \& Hollingshead, 2011). In order to facilitate team learning and foster a sense of psychological safety, ICU team leaders must talk openly about mistakes and difficulties in order to create a culture where there is no fear of retribution for clear and candid communication about potential problems (e.g., Edmonson, 2012).

\section{Team Performance in the Intensive Care Unit}

The goals of ICU teams are multifold. In most published research, patient outcomes in general, and mortality rates specifically, are the most common metric of ICU team performance. Although mortality rates are a commonly used performance metric, they are 
not necessarily the best indicator of success considering that end-of-life care is a primary treatment administered in the ICU. The goal of end-of-life-care is to agree upon desired outcomes and achieve realistic goals of care; yet many of these decisions are complex (i.e., many factors must be considered by families and care providers) and sometimes even controversial, which can complicate coordination of care (e.g., Cook \& Rocker, 2014).

Thus, mortality should not be the only indicator for determining successful ICU team performance. Other goals include but are not limited to improving health-related quality of life among ICU survivors, improving the quality of death and dying among ICU decedents, acting as efficient stewards for health care resources by avoiding waste, and tending to the needs of family members with loved ones in the ICU. However, understanding team performance is complicated by the fact that these goals do not always align. For example, heroic efforts to save lives are costly, necessitating time and expensive technology. In contrast, early deaths are inexpensive. In this way, an ICU team with high performance along the domain of mortality can be a low performer along the domain of costs. These tensions have several implications for how we understand ICU team performance. First, it is difficult to characterize effective teams, in that high performance along one domain does not necessarily translate to high performance along another domain. The challenge for efforts to improve ICU teamwork, then, is to identify not only what kinds of processes or interventions could improve specific outcomes, but also to elucidate boundary conditions and unintended negative consequences of different practices. Second, it demonstrates the need for ICU teams themselves to define their goals and agree upon their prioritizations. Goal priorities will vary from patient to patient within a single day and over time, and to the degree that ICU teams do not a priori agree on the goals for a given patient, team performance can never be optimized.

Acknowledging the issue of competing goals, in the following sections we review the literature on ICU team performance with a focus on risk-adjusted mortality rates, as mortality is by far the most commonly employed performance metric in the literature. For clarity, we also focus on studies examining adult general, non-specialty critical care, although the issues we discuss also apply to specialty units such as neonatal ICUs, pediatric ICUs, and neurological ICUs.

Team Composition-ICU teams are typically composed of an intensivist physician, a clinical pharmacist, a dietician, several respiratory therapists and bedside nurses, and other health care providers such as clinical psychologists. The intensivist, a physician with specialized training in critical care medicine, is the leader of the team and has ultimate responsibility for medical decision-making. An extensive body of literature demonstrates that the presence of an intensivist as team leader as opposed to a physician without specialty critical care training, is associated with lower mortality (Wilcox et al., 2013), yet little is known about the underlying reasons for this observation. The conventional wisdom is that intensivists bring experience and expertise in the care of critically ill patients that nonintensivist physicians do not possess, which may lead to improve leadership skills and improved team performance (Cooke et al., 2008; Kahn, Brake, \& Stenberg, 2007). 
Current professional guidelines recommend intensivist-led care for all ICU patients, although not all ICUs in the United States conform to this standard (Weled et al., 2015). One reason for such variability is that over the past decade the interprofessional care model has also become more prevalent (Kohn et al., 2017). Patient care under this model relies on collaborations from experts from various domains (e.g., respiratory therapy, clinical pharmacy, critical care nursing, clinical psychology) in the ICU. With the increasing popularity of the interprofessional care model, the value of an intensivist appears to be decreasing, as interprofessional providers have gained expertise and are able to take a more active role in caring for patients, thus diffusing medical decision-making responsibilities across members of the care team. In addition, the increased use of protocols and other communication tools has lessened the need for intensivist-led care (Costa, Wallace, \& Kahn, 2015; Kohn et al., 2017; Wilcox et al., 2014; Yoo, Edwards, Dean, \& Dudley, 2014).

Clinical pharmacists provide unique expertise on drugs that are the cornerstone of ICU treatment. Drug administration and dosages can be highly influential on patient outcomes, and intensivist physicians might not possess all of this information at the ready. Data demonstrate that the presence of a clinical pharmacist in the ICU is associated with lower adverse drug events and improved patient outcomes (Kane, Weber, \& Drasta, 2003; Leape et al., 1999; Rivkin \& Yin, 2011; Stone et al., 2011).

Dieticians provide unique expertise for patients' nutritional needs, and must account for the problem that feeding protocols are often contingent upon other therapies (Cahill, Dhaliwal, Day, Jiang, \& Heyland, 2010). For example, when and how patients are provided nutritional support is in part determined by whether they are placed on a ventilator, their level of alertness, and their immune system functioning. Therefore, dieticians must collaborate with all members of the ICU team to ensure that patients receive adequate and timely nutritional support.

Respiratory therapists typically oversee the provision of mechanical ventilation, which is the central supportive therapy for patients experiencing respiratory failure and among the most common ICU treatments (Netzer et al., 2011; Stamm, 2005). Although the provision of mechanical ventilation is collaborative by nature, respiratory therapists possess unique expertise and experience in how to operate the ventilator, and, like pharmacists, their involvement in care is associated with lower mortality in the ICU (Ely et al., 1996).

Nurses are, among other things, responsible for closely monitoring and reporting changes in patients' health and wellbeing (Baggs et al., 1999; Knoll \& Lendner, 2008). Unlike other members of the ICU team who care for most if not all patients at any given time, nurses only care for a subset of patients, typically no more than two at any one time. Yet nurses are central members of the ICU team because they are directly involved in nearly all ICU treatments, in that they are responsible for assessing vital signs, delivering drugs, and monitoring for complications of therapy. Greater nursing education and expertise is associated with lower mortality among ICU patients (Kelly, Kutney-Lee, McHugh, Sloane, \& Aiken, 2014). 
When available and able to join the care team, clinical psychologists and other behavioral healthcare specialists play a unique role in that they specifically address patients' psychological recovery and they provide care for patients, families, and critical care providers. Particularly noteworthy is that patients who receive care by a clinical psychologist have lower rates of anxiety, depression, and post-traumatic stress following admission to the ICU (Nova \& Ballesteros de Valderama, 2006; Peris et al., 2011).

Some team members might be in training to become critical care providers, and may come from any of the above disciplines. Most common are physicians-in-training (i.e., medical students, interns, residents, and fellows), and advanced practice providers (APPs) who are nurse practitioners and physician assistants being trained to perform many of the same tasks as an attending physician. The presence of clinicians-in-training is positively associated with role clarity, as well as the frequency and quality of information exchanges among ICU team members (Hawryluck, Espin, Garwood, Evans, \& Lingard, 2002; Joffe, Pastores, Maerz, Mathur, \& Lisco, 2014; Valentin \& Ferinande, 2011, but see Almoosa, Goldenhar, Puchalski, Ying, \& Panos, 2010; Costa, Wallace, Barnato, \& Kahn, 2014). And, while there has been some concern that reliance on nurse practitioners and physician assistants in training might negatively impact patient outcomes, findings suggest that this is not the case (e.g., Costa et al., 2014).

Each of the clinician types possesses a diverse array of knowledge acquired through different training pathways. In turn, each possesses unique skills, jargon, and status within the team (Alexanian et al., 2015; Azoulay et al., 2009; Ferrand et al., 2003; Kho, Carbone, Lucas, \& Cook, 2005). These differences can improve the quality of care but can also lead to ineffective interprofessional interactions and conflict among the clinicians. These interprofessional conflicts tend to be multifactorial, making them difficult to solve. Importantly, unresolved conflict can impede open exchanges of information and reduce appreciation of one another's expertise (Mitchell, Parker, Giles, \& Boyle, 2014; Wright, Bowkett, \& Bray, 1996), which delays and reduces the quality of patient care (Azoulay et al., 2009; Lindgard, Epsin, Evans, \& Hawryluck, 2004; Ten Have \& Nap, 2014; Wysham et al., 2017).

In the modern ICU, family members are increasingly considered to be part of the ICU team, especially when they take on the role of surrogate decision makers for loved ones who are too ill to advocate for themselves. The practice of family participation on medical rounds has received the most attention in this area, with data suggesting that while rounds are traditionally viewed as a forum for collaboration among clinicians, they can also be used for collaboration with family members, including information exchanges and reducing decisional conflict (Davidson et al., 2017). However, some clinicians feel that family presence can disrupt important communication processes (Jacobowsky, Girard, Mulder, \& Ely, 2010; Reeves, et al., 2015; Santiago, Lazar, Jiang, \& Burns, 2014). For instance, clinicians have expressed reluctance to engage in candid discussions about patient prognoses while in the presence of family members (Au, des Ordons, Soo, Guienguere, \& Stelfox, 2016; Azoulay et al., 2009; Huffines et al. 2013; Maxwell et al., 2007). 
Team Collaboration and Decision Making-The foundation of ICU team collaboration and decision-making is daily rounds. Rounds are the formal, daily face-to-face meetings that are attended by most if not all of the ICU clinicians that are directly involved with patient care (Hawryluck et al., 2002). On rounds each ICU patient is discussed either at the bedside or in another area such as a hallway or conference room. As a conduit for team processes, rounds provide a context for critical care providers to share information and engage in shared decision-making processes. Rounds also serve as a platform for clinicians to share their experiences and advice concerning critical care more broadly, such as sensitive issues regarding patient and family communication, strategies for dealing with difficult situations, and to provide other forms of psychosocial support (Lown \& Manning, 2010). Rounds are typically structured such that clinicians work systematically through all of the patients admitted to the ICU on a given day, meaning that as the team moves through the ICU, team member rotate in and out of the discussion such that the composition of the team varies from patient to patient.

Due to their ephemeral nature, collaborative information sharing during rounds is a difficult endeavor. Effective rounds allow clinicians to openly exchange information about patient care, which should result in fewer knowledge- and/or training-related errors (Hawryluck et al., 2002; Kim, Barnato, Angus, Fleisher, \& Kahn, 2010; Montague, Lee, \& Hussain, 2004). Yet rounds are frequently interrupted by phone calls and clinical emergencies, which disrupts flow and increases the amount of time clinicians spend during rounds, thereby limiting the time left in the day to care for patients or communicate with family members (Alverez \& Coicera, 2005; Fackler, Watts, Grome, Miller, Crandall, \& Pronovost, 2009; Giri et al., 2013; Hawryluck et al., 2002; Ward, Read, Afessa, \& Kahn, 2012). Clinicians will often compensate for anticipated interruptions by withholding information during rounds to speed up the process, which can increase the efficiency yet the decrease the effectiveness of rounds as a communication tool (Costa et al., 2014).

While rounds represent formal communication procedures in the ICU, informal information sharing and decision making will often take place between small subsets of members of the team throughout the day (Alexanian et al., 2015; Costa et al., 2014). These types of informal interactions are particularly prevalent when (a) rounds are not conducted in a timely manner, (b) family member presence makes clinicians reticent to discuss negative patient prognoses or other sensitive information, and (c) clinicians are unsure of their positions and/or lack the psychological safety needed to speak up in the presence of other team members during rounds. While informal discussions cannot be avoided altogether, they are problematic in that they perpetuate information gaps among care providers.

Checklists and Protocols-To overcome barriers to effective formal and informal collaboration, many ICUs have developed communication and decision-making strategies to facilitate team functioning and performance. For example, many ICUs have implemented checklists or daily goal forms to promote effective communication among clinicians (Gawande, 2009). These tools systematize the ways that clinicians discuss key treatments, and provide structure and guidelines for interactions that should establish shared goals of care and clarify clinicians' roles. Checklists and protocols are meant to streamline care, which is an important consideration in that many critical care providers report time 
constraints as one of the biggest challenges to providing high quality care (Azoulay et al., 2009; Dodek \& Rabound, 2013; Narasimhan, Eisen, Mahoney, Acerra, \& Rosen, 2006; Idahosa \& Kahn, 2002; Pronovost et al., 2003; Ward et al., 2013; Weiss et al., 2011). However, implementation of these tools can be time consuming and reduce complex decisions to rote processes, which can lead to fatigue. These types of protocols also do consistently improve patient outcomes (Writing Group for the CHECKLIST-ICU Investigators and the Brazilian Research in Intensive Care Network, 2017).

Other Determinants of ICU Team Performance-ICU team performance is determined not only by effective coordination and conflict management, but also by organizational and environmental characteristics exogenous to team processes and protocols. As suggested by the existing literature, team formation and rounding processes appear to be the most consequential factors to consider for influencing team performance, and therefore the likely levers for change to be targeted by future interventions.

First, with regard to staffing decisions, our takeaway from the research is that it is not necessarily whether an ICU has an intensivist present that is at issue, but rather the extent to which a team leader is able to help overcome barriers to effective communication that are inherent in interprofessional and interdisciplinary teams. Having an intensivist lead the ICU team might help mitigate problems associated with status differences and jargon unique to a clinician's specialty, and to provide role and goal clarity (Hawryluck et al., 2002; Kim et al., 2010), but there may be other ways to address these processes that do not require an intensivist on the team.

Second, rounding processes represent the time when ICU teams are most "team like," in that most members are present and have the option to speak up during rounds, and shared goals can be established and/or maintained. As such, we believe that practices that facilitate effective problem solving and the establishment of psychological safety during rounds will have positive carryover effects as team members interact throughout the day. Thus, interventions aimed at improving ICU team functioning should target team-level behaviors that influence communication, conflict, and group decision-making during rounds.

\section{Future Directions}

Improving ICU teams will require research and quality improvement efforts that draw directly from the team science literature, which can provide relevant theories and concepts that will elicit a better understanding of whether and how various psychosocial factors develop and influence team effectiveness over time. Extant studies predominantly focus on leadership and psychological safety, but many other theories and concepts potentially apply to ICU teams. For example, conflict can have either positive or negative effects on team performance depending on situational and team factors (De Dreu \& Weingart, 2003; de Wit, Greer, \& Jehn, 2012). Yet little is known beyond the fact that (a) nurses and intensivists report engaging in the most conflict (e.g., Azoulay et al., 2009), and (b) nurses engage in a fair amount of (negative and sometimes abusive) conflict with other nurses (Alspach, 2007). Future work on ICU teams should examine different sources of conflict (Cronin \& Weingart, 2007) as well as the frequency, intensity, and expression of different types of conflict 
(Weingart, Behfar, Bendersky, Todorova, \& Jehn, 2015) in order to determine more effective ways to manage conflict in the ICU.

Another issue that currently lacks resolution is how precisely to define an ICU "team." At one extreme, the most inclusive characterization would define the team as all ICU staff, to include administrators and those only indirectly involved with patient care. However, such a definition ignores important interdependencies among clinicians who work together to provide direct care for patients, and discounts temporal dynamics related to team composition (Shortell et al., 1994). At the other extreme, a minimal definition would characterize an ICU team as two clinicians working together to provide a given treatment to a given patient at a particular point in time. By this definition, though, ICU teams are probably too transitory to warrant attempts to make generalizations across teams or ICUs. Future research should consider linking daily team composition (of those directly involved with the care of a given patient) and related dynamics to quality care indices and patient outcomes over time. The literature on multiple team membership could inform this research (Marks, DeChurch, Mathieu, Panzer, \& Alonso, 2005; O'leary, Mortensen, \& Woolley, 2011) and make for an exciting new research direction that informs our understanding of ICU team functioning and multiple team membership more generally. An alternative but equally fruitful approach could apply network analysis to evaluate dynamics of critical care providers and ICU teams as parts of a larger multi-team system (Poole and Contractor, 2012).

Finally, efforts to reconcile the varying and often competing measures of ICU team effectiveness and performance is needed. Considering the increased reliance on team-based care in the ICU, it is paramount that we continue to elucidate connections between the team processes and patient-, family-, ICU-, and hospital-level outcomes. Moreover, risk-adjusted mortality alone does not allow the identification of how to leverage the team when accounting for the varying and sometimes contradictory goals of these stakeholders. Reader et al. (2009) offered a model to categorize and test the impact of team inputs and processes on different ICU team outcomes. An adapted version of this model can help account for different ways the team will influence and can produce optimal levels of patient satisfaction and quality care, or lower staff burnout and turnover, health care costs, or one of the many other potential outcomes of interest.

For the most part we have refrained from commenting on interventions, as thorough reviews on medical teams are available elsewhere (e.g., Hughes et al., 2016), and we are somewhat agnostic as to whether unique features of the ICU enable the generalization of that work to teams in this domain. Furthermore, most of the critical care research to date is focused primarily on academic medical centers. Due to idiosyncrasies and differences across community samples, we also know little about how teamwork might operate differently within these settings. Nevertheless, the ultimate goal we share with others is to understand how to provide better team-based care and intervene with informed evidence-based practices when necessary.

In the meantime, both clinical psychologists and researchers in the field of psychology can approach the ICU as an opportunity to extend our understanding of team functioning in 
health care, one in which the existing research demonstrates the importance of role clarity, psychological safety, and leader inclusiveness in teams that are both highly hierarchical and low in temporal stability. With extremely high stakes, not only for patients at risk of death and disability but also family members and providers at risk for psychological distress and burnout, the team is likely to play an increasingly vital role in ensuring the ICU meets its goal of saving lives by ensuring that critically ill patients receive the best care possible.

\section{References}

Alameddine M, Dainty KN, Deber R, \& Sibbald WJB (2009). The intensive care unit work environment: Current challenges and recommendations for the future. Journal of Critical Care, 24 , 243-248. doi:10.1016/j.jcrc.2008.03.038 [PubMed: 19327295]

Alexanian JA, Kitto S, Rak KJ, \& Reeves S (2015). Beyond the team: Understanding interprofessional work in two North American ICUs. Critical Care Medicine, 43, 1880-1886. doi:1097/CCM. 00000000000000136 [PubMed: 26102250]

Almoosa KF, Goldenhar LM, Puchalski J, Ying J, \& Panos RJ (2010). Critical acre education during internal medicine residency: A national survey. Journal of Graduate Medicine Education, 2, 555561. doi:10.4300/JGME-D-10-00023.1

Alspach G (2007). Critical care nurses as coworkers: Are our interactions nice or nasty? Critical Care Nurse, 27, 10-14.

Alvarez G, \& Coiera E (2005). Interruptive communication patterns in the intensive care unit ward round. International Journal of Medical Informatics, 74, 791-796. doi:10.1016/j.ijmedinf. 2005.03.017 [PubMed: 16024286]

Andreatta PB (2010). A typology for health care teams. Health Care Management Review, 35, 345354. doi:10.1097/HMR.0b013d3181e9fceb [PubMed: 20844359]

Au SS, des Ordons AR, Soo A, Guienguere S, \& Stelfox HT (2016). Family participation in intensive care unit rounds: Comparing family and provider perspectives. Journal of Critical Care, 38, 132136. doi:10.1016/j.jcrc.2016.10.020 [PubMed: 27888716]

Azoulay E, Timsit JF, Sprung CL, Soares M, Rusinová K, Lafabrie A, .. \& Benoit D (2009). Prevalence and factors of intensive care unit conflicts: The conflicus study. American Journal of Respiratory and Critical Care Medicine, 180, 853-860. doi:10.1164/rccm.2008010-1614OC [PubMed: 19644049]

Baggs JG, Schmitt MH, Mushlin AI, Mitchell PH, Eldredge DH, Oakes D, \& Hutson AD (1999). Association between nurse-physician collaboration and patient outcomes in three intensive care units. Critical Care Medicine, 27, 1991-1998. doi:10.1097/00003246-199909000-00045 [PubMed: 10507630]

Barrett ML, Smith MW, Elixhauser A, Honigman LS, \& Pines JM (2011). Utilization of intensive care services. Healthcare Cost and Utilization Project. Retrieved from http://hcup-us.ahrq.gov/reports/ statbriefs/sb185-Hospital-Intensive-Care-Units-2011.jsp

Cahill NE, Dhaliwal R, Day AG, Jiang X, \& Heyland DK (2010). Nutrition therapy in the critical care setting: what is "best achievable" practice? An international multicenter observational study. Critical Care Medicine, 38, 395-401. doi:10.1097/CCM.0b013e3181c0263d [PubMed: 19851094]

Cook D, \& Rocker G (2014). Dying with dignity in the intensive care unit. The New England Journal of Medicine, 370, 2506-2514. doi:10.1056/NEJMra1208759 [PubMed: 24963569]

Cooke CR, Watkins TR, Kahn JM, Treggiari MM, Caldwell E, Hudson LD, \& Rubenfeld GD (2008). The effect of an intensive care unit staffing model on tidal volume in patients with acute lung injury. Critical Care, 12, R134. [PubMed: 18980682]

Costa DK, Wallace DJ, Barnato AE, \& Kahn JM (2014). Nurse practitioner/physician assistant staffing and critical care mortality. Chest, 146, 1566-1573. doi: 10.1056/NEJMsa1201918 [PubMed: 25167081]

Costa DK, Wallace DJ, \& Kahn JM (2015). The association between daytime intensivist physician staffing and mortality in the context of other ICU organizational practices: A multicenter cohort 
study. Critical Care Medicine, 43, 2275-2282. doi:10/1007/CCM.0000000000001259 [PubMed: 26308426]

Cronin MA, \& Weingart LR (2007). Representational gaps, information processing, and conflict in functionally diverse teams. Academy of Management Review, 32, 761-773. doi:10.5465/AMR. 2007.25275511

Curtis JR, Cook DJ, Wall RJ, Angus DC, Bion J, Kacmarek R, .. \& \& Moreno R (2006). Intensive care unit quality improvement: A "how-to" guide for the interdisciplinary team. Critical Care Medicine, 34, 211-218. doi:10.1097/01.CCM.0000190617.76104.AC [PubMed: 16374176]

Davidson JE, Aslakson RA, Long AC, Puntillo KA ... \& Randall CJ (2017). Guidelines for familycentered care in the neonatal, pediatric, and adult ICU. Critical Care Medicine, 45, 103-128. doi: 10.1097/CCM.00000000000002169 [PubMed: 27984278]

De Dreu CKW, \& Weingart LR (2003). Task versus relationship conflict, team performance and team member satisfaction: A meta-analysis. Journal of Applied Psychology, 88, 741-749. doi: 10.1037/0021-9010.88.4.741 [PubMed: 12940412]

de Wit FRC, Greer LL, \& Jehn KA (2012). The paradox of intragroup conflict: A meta-analysis. Journal of Applied Psychology, 97, 360-390. doi:10.1037/a0024844 [PubMed: 21842974]

Dodek PM, \& Raboud J (2003). Explicit approach to rounds in an ICU improves communication and satisfaction of providers. Intensive Care Medicine, 29, 1584-1588. doi:10.1007/s00134-003-1815y [PubMed: 12898001]

Durbin CG (2006). Team model: Advocating for the optimal method of care delivery in the intensive care unit. Critical Care Medicine, 34, S12-S17. doi:1097/01.CCM.0000199985.72497.D1 [PubMed: 16477198]

Edmondson AC (2012). Teaming: How organizations learn, innovate, and compete in the knowledge economy. Hoboken, NJ: John Wiley \& Sons.

Ely EW, Baker AM, Dunagan DP, Burke HL, Smith AC, Kelly PT, ... \& Haponik EF (1996). Effect on the duration of mechanical ventilation of identifying patients capable of breathing spontaneously. The New England Journal of Medicine, 335, 1864-1869. 10.1056/NEJM199612193352502 [PubMed: 8948561]

Embriaco N, Papazian L, Kentish-Barnes N, Pochard F, \& Azoulay E (2007). Burnout syndrome among critical care healthcare workers. Current Opinion in Critical Care, 13, 482-488. doi: 10.1097/MCC.0b013e3282efd28a [PubMed: 17762223]

Fackler JC, Watts C, Grome A, Miller T, Crandall B, \& Pronovost P (2009). Critical care physician cognitive task analysis: an exploratory study. Critical Care, 13, 1. doi:10.1186/cc7740

Fernandez R, \& Grand JA (2015). Leveraging social science-healthcare collaborations to improve teamwork and patient safety. Pediatric and Adolescent Health Care, 45, 370-377. doi:10.1016/ cppeds.2015.10.005 [PubMed: 26573242]

Ferrand E, Lemaire F, Regnier B, Kuteifan K, Badet M, Asfar P, .. \& Pochard F (2003). Discrepancies between perceptions by physicians and nursing staff of intensive care unit end-of-life decisions. American Journal of Respiratory and Critical Care Medicine, 167, 1310-1315.doi:10.1164/rccm. 200207-752OC [PubMed: 12738597]

Gawande A (2009). The checklist manifesto. Henry Holt and Company, New York.

Giri J, Ahmed A, Dong Y, Keegan MT, Herasevich V, Gajic O, \& Pickering B (2013). Daily intensive care unit rounds: A multidisciplinary perspective. Applied Medical Informatics, 33, 63-73.

Hawryluck LA, Espin SL, Garwood KC, Evans CA, \& Lingard LA (2002). Pulling together and pushing apart: tides of tension in the ICU team. Academic Medicine, 77, S73-S76. [PubMed: 12377711]

Henrich NM, Dodek PH, Alden L, Keenan SP, Reynolds S, \& Rodney P (2016). Causes of moral distress in the intensive care unit: A qualitative study. Journal of Critical Care, 35, 57-62. doi: 10.1016/j.jcrc.2016.04.033 [PubMed: 27481736]

Hoover R (2017). Benefits of using an electronic health record. Nursing Critical Care, 12, 9-10. doi: 10.1097/01.CCN.0000508631/93151.8d

Huffines M, Johnson KL, Naranjo LLS, Lissauer ME, Fishel MAM, Howes SMA, ... \& Smith R (2013). Improving family satisfaction and participation in decision making in an intensive care unit. Critical Care Nurse, 33, 56-69. doi:10.4037/ccn2012254 [PubMed: 24085828] 
Hughes AM, Gregory ME, Joseph DL, Sonesh SC, Marlow SL, Lacerenza CN, ... \& Salas E (2016). Saving lives: A meta-analysis of team training in healthcare. Journal of Applied Psychology, 101, 1266-1304. doi:10.1037/apl0000120. [PubMed: 27599089]

Idahosa O, \& Kahn JM (2012). Beyond checklists: Using clinician prompts to achieve meaningful ICU quality improvement. Critical Care, 16, 1. doi:10.1186/cc11199

Jacobowski NL, Girard TD, Mulder JA, \& Ely EW (2010). Communication in critical care: Family rounds in the intensive care unit. American Journal of Critical Care, 19, 421-430. doi:10.4037/ ajcc2010656kjln [PubMed: 20810417]

Joffee AM, Pastores SM, Maerz LL, Mathur P, \& Lisco SJ (2014). Utilization and impact on fellowship training of non-physician advanced practice providers in intensive care units of academic medical centers: A survey of critical care program directors. Journal of Critical Care, 29, 112-115. [PubMed: 24268625]

Kahn JM, Brake H, \& Steinberg KP (2007). Intensivist physician staffing and the process of care in academic medical centres. Quality and Safety in Health Care, 16, 329-333. doi:10.1136/qshc. 2007.022376 [PubMed: 17913772]

Kane SL, Weber RJ, \& Dasta JF (2003). The impact of critical care pharmacists on enhancing patient outcomes. Intensive Care Medicine, 29, 691-698. doi:10.1007/s00134-003-1705-3 [PubMed: 12665997]

Kelly DM, Kutney-Lee A, McHugh MD, Sloane DM, \& Aiken LH (2014). Impact of critical care nursing on 30-day mortality of mechanically ventilated older adults. Critical Care Medicine, 42, 1089-1095. [PubMed: 24368346]

Kesecioglu J, Schneider MM, van der Kooi AW, \& Bion J (2012). Structure and function: planning a new ICU to optimize patient care. Current Opinion in Critical Care, 18, 688-692. doi:1097/MCC. 0b013e328358d4bd [PubMed: 23010634]

Kim MM, Bernato AE, Angus DC, Fleisher LF, \& Kahn JM (2010). The effect of multidisciplinary care teams on intensive care unit mortality. Archives of Internal Medicine, 22, 369-376. doi: 10.1001/archintermed.2009.521

Kho ME, Carbone JM, Lucas J, \& Cook DJ (2005). Safety Climate Survey: Reliability of results from a multicenter ICU survey. Quality and Safety in Health Care, 14, 273-278. doi:10.1136/qshc. 2005.014316 [PubMed: 16076792]

Knoll M, \& Lendner I (2008). [Nurses' perspective on interprofessional communication on an intensive care unit]. Pflege, 21, 339-351. doi:10.1024/1012-5302.21.5.339 [PubMed: 18850538]

Kohn R, Madden V, Kahn JM, Asch DA, Barnato AE, Halpern SD, \& Kerlin MP (2017). Diffusion of evidence-based intensive care unit organizational practices. A state-wide analysis. Annals of the American Thoracic Society, 14, 254-261. doi:10.1513/AnnalsATS.201607-5790C [PubMed: 28076685]

Leape LL, Cullen DJ, Clapp MD, Burdick E, Demonaco HJ, Erickson JI, \& Bates DW (1999). Pharmacist participation on physician rounds and adverse drug events in the intensive care unit. JAMA, 282, 267-270. doi:10.1001/jama.282.3.267 [PubMed: 10422996]

Lingard L, Espin S, Evans C, \& Hawryluck L (2004). The rules of the game: Interprofessional collaboration on the intensive care unit team. Critical Care, 8, 1. doi: 10.1186/cc2958 [PubMed: 14975035]

Lown BA, \& Manning CF (2010). The Schwartz Center Rounds: Evaluation of an interdisciplinary approach to enhancing patient-centered communication, teamwork, and provider support. Academic Medicine, 85, 1073-1081. doi:10.1097/ACM.0b013e3181dbf741 [PubMed: 20505412]

Manthous CA, \& Hollingshead AB (2011). Team science and critical care. American Journal of Respiratory and Critical Care Medicine, 184, 17-25. doi:101164/rccm.201101-0185CI [PubMed: 21471081]

Manthous CA, Nembhard IM, \& Hollingshead AB (2011). Building effective critical care teams. Critical Care, 15, 307-313. doi:10.1186/cc10255 [PubMed: 21884639]

Marks MA, DeChurch LA, Mathieu JE; Panzer FJ, \& Alonso A (2005). Teamwork in multiteam systems. Journal of Applied Psychology, 90, 964-971. doi:10.1037/0021-9010.90.5.964 [PubMed: 16162068] 
Maxwell KE, Stuenkel D, \& Saylor C (2007). Needs of family members of critically ill patients: A comparison of nurse and family perceptions. Heart \& Lung: The Journal of Acute and Critical Care, 36, 367-376. doi:10.1016/j.hrting.2007.02.005 [PubMed: 17845883]

Mitchell R, Parker V, Giles M, \& Boyle B (2014). The ABC of health care team dynamics: Understanding complex affective, behavioral, and cognitive dynamics in interprofessional teams. Health Care Management Review, 39, 1-9. doi:10.1097/HCM.0b013e3182766504 [PubMed: 24304597]

Montague ML, Lee MSW, \& Hussain SSM (2004). Staff attitudes to a daily otolaryngology ward round. The Journal of Laryngology \& Otology, 118, 963-971.doi:10.1258/0022215042790682 [PubMed: 15667684]

Narasimhan M, Eisen LA, Mahoney CD, Acerra FL, \& Rosen MJ (2006). Improving nurse-physician communication and satisfaction in the intensive care unit with a daily goals worksheet. American Journal of Critical Care, 15, 217-222. doi:10.1378/chest_126_4 [PubMed: 16501141]

Netzer G, Liu X, Shanholtz C, Harris A, Verceles A, \& Iwashyna TJ (2011). Decreased mortality resulting from a multicomponent intervention in a tertiary care medical intensive care unit. Critical Care Medicine, 39, 284-293. doi:10.1097/CCM.0b013e3181ffdd2f [PubMed: 21076286]

Novoa M, \& Ballesteros de Valderrama BP (2006). The role of the psychologist in an intensive care unit. Universitats Psychologica, 5, 599-612.

O'leary MB, Mortensen M \& Woolley AW (2011). Multiple team membership: A theoretical model of its effects on productivity and learning for individuals and teams. Academy of Management Review, 36, 461-478. doi: 10.5465/amr.2009.0275

Ong MS, BiomedE M, \& Coiera E (2011). A systematic review of failures in handoff communication during intrahospital transfers. The Joint Commission Journal on Quality and Patient Safety, 37, 274-284. doi:10/1016/S1553-7250(11)37035-3 [PubMed: 21706987]

Patterson ES, Roth EM, Woods DD, Chow R, \& Gomes JO (2004). Handoff strategies in settings with high consequences for failure: lessons for health care operations. Interantional Journal for Quality in Health Care, 16, 125-132. doi:10.1093/intqhe/mzh026

Peris A, Bonizzolo M, Iozzelli D, Migliaccio ML\& Belloni L (2011). Early intra-intensive care unit psychological intervention promotes recovery from post traumatic stress disorders, anxiety and depression symptoms in critically ill patients. Critical Care, 15, 1-8. doi:10/1186/cc10003

Piquette D, Reeves S, \& LeBlanc VR (2009). Stressful intensive care unit medical crises: How individual responses impact on team performance. Critical Care Medicine, 37, 1251-1255. doi: 10.1097/CCM.0b013e31819c1496 [PubMed: 19242320]

Poole MS \& Contractor N (2012). Conceptualizing the multi-team system as ecosystem of networked groups In Zaccaro S, Marks M, \& DeChurch L (Eds.) Multi-team systems (pp. 193-224). New York: Routledge.

Pronovost P, Berenholtz S, Dorman T, Lipsett PA, Simmonds T, \& Haraden C (2003). Improving communication in the ICU using daily goals. Journal of Critical Care, 18, 71-75. doi:10.1053/jcrc. 2003.50008 [PubMed: 12800116]

Rashid M (2006). A decade of adult intensive care unit design: A study of the physical design features of the best practice examples. Critical Care Nursing Quarterly, 29, 282-311. [PubMed: 17063097]

Reader TW, Flin R, Mearns K, \& Cuthbertson BH (2009). Developing a team performance framework for the intensive care unit. Critical Care Medicine, 37, 1787-1793. doi:10.1097/CCM. 0b013e31819f0451. [PubMed: 19325474]

Reeves S, McMillan SE, Kachan N, Paradis E, Leslie M, \& Kitto S (2015). Interprofessional collaboration and family member involvement in intensive care units: Emerging themes from a multi-sited ethnography. Journal of Interprofessional Care, 29, 230-237. doi: 10.3109/13561820.2014.955914 [PubMed: 25238573]

Rivkin A, \& Yin H (2011). Evaluation of the role of the critical care pharmacist in identifying and avoiding or minimizing significant drug-drug interactions in medical intensive care patients. Journal of Critical Care, 26, 104.e1-104.e6. doi:10.1016/j.jcrc.2010.0r4.014

Salas E, Cooke NJ, \& Rosen MA (2008). On teams, teamwork, and team performance: Discoveries and developments. Human Factors: The Journal of the Human Factors and Ergonomics Society, 50, 540-547. doi:10.1518/001872008X288457 
Salas E, Dickson TL, Convese SA, \& Tannenbaum SI (1992). Toward an understanding of team performance and training In Swezey RW \& Salas E (Eds.), Their training and performance (pp. 329). Norwood, NJ: Ables.

Salas E, Shuffler ML, Thayer AL, Bedwell WL, \& Lazzara EH (2015). Understanding and improving teamwork in organizations: A scientifically based practice guide. Human Resource Management, 54, 599-622. doi:10.1002/hrm.21628

Santiago C, Lazar L, Jiang D, \& Burns KEA (2014). A survey of the attitudes and perceptions of multidisciplinary team members towards family presence at bedside rounds in the intensive care unit. Intensive and Critical Care Nursing, 30, 13-21. doi:10.1016/j.iccn.2013.06.003 [PubMed: 23962578]

Shortell SM, Zimmerman JE, Rousseau DM, Gillies RR, Wagner DP, Draper EA, ... \& Duffy J (1994). The performance of intensive care units: does good management make a difference?. Medical Care, 32, 508-525. [PubMed: 8182978]

Stamm AM (1998). Ventilator-associated pneumonia and frequency of circuit changes. American Journal of Infection Control, 26, 71-73. doi:10.1016/SO196-6553(98)70064-1 [PubMed: 9503116]

Stone ME, Snetman D, O’Neill A, Cucuzzo J, Lindner J, Ahmad S, \& Teperman S (2011). Daily multidisciplinary rounds to implement the ventilator bundle decreases ventilator-associated pneumonia in trauma patients: but does it affect outcome?. Surgical Infections, 12, 373-378. doi: 10.1089/sur.2010.067 [PubMed: 21933008]

Ten Have ECM, \& Nap RE (2014). Mutual agreement between providers in intensive care medicine on patient care after interdisciplinary rounds. Journal of Intensive Care Medicine, 29, 292-297. doi: 10.1177/0885066613486596 [PubMed: 23753243]

Thomas EJ, Sexton BJ, \& Helmreich RL (2003). Discrepant attitudes about teamwork among critical care nurses and physicians. Critical Care Medicine, 31, 956-959. doi:10.1097/01.CCM. 0000000056183.89175.76 [PubMed: 12627011]

Thompson DR, Hamilton DK, Cadenhead CD, Swoboda SM, Schwindel SM, Anderson DC, .... Peterson C (2012). Unit design guidelines for intensive care. Critical Care Medicine, 40, 15861600. doi:10.1097/CCM.0b013e3182413bb2 [PubMed: 22511137]

Valentin A, \& Ferdinande P (2011). Recommendations on basic requirements for intensive care units: Structural and organizational aspects. Intensive Care Medicine, 37, 1575-1587. doi:10.1007/ s00134-011-2300-7. [PubMed: 21918847]

Ward NS, Afessa B, Kleinpell R, Tisherman S, Ries M, Howell M, Halpern N, \& Kahn J (2013). Intensivist/patient ratios in closed ICUs: A statement from the Society of Critical Care Medicine Taskforce on ICU staffing. Critical Care Medicine, 41, 638-645. doi:10/1097/CCM. Ob013e3182741478 [PubMed: 23263586]

Ward NS, Read R, Afessa B, \& Kahn JM (2012). Perceived effects of attending physician workload in academic medical intensive care units: A national survey of training program directors. Critical Care Medicine, 40, 400-405. doi:10.1097/CCM.0b013e318232d997 [PubMed: 22001582]

Weingart LR, Behfar KJ, Bendersky C, Todorova G, \& Jehn KA (2015). The directness and oppositional intensity of conflict expression. Academy of Management Review, 40, 235-262. doi: 10.5465/amr.2013.0124

Weiss CH, Moazed F, McEvoy CA, Singer BD, Szleifer I, Amaral LA, .. \& S Sznajder JI (2011). Prompting physicians to address a daily checklist and process of care and clinical outcomes: a single-site study. American Journal of Respiratory and Critical Care Medicine, 184, 680. doi: 10.1164/rccm.201101-003OC [PubMed: 21616996]

Weled BJ, Adzhigirey LA, Hodgman TM, Brilli RJ, Spevetz A, Kline AM, ... Wheeler DS (2015). Critical care delivery: The importance of process of care and ICU structure to improved outcomes, 43, 1520-1525. doi:10.1097/CCM.00000000000000000978

Wilcox ME, Chong CAKY, Niven DJ, Rubenfeld GD, Rowan KM, Wunsch H, \& Fan E (2013). Do intensivist staffing patterns influence hospital mortality following ICU admission? A systematic review and meta-analyses, 41, 2253-2274. 
Wildman JL, Shuffler ML, Lazzara EH, Fiore SM, Burke CS, Salas E, \& Garven S (2012). Trust development in swift starting action teams: A multilevel framework. Group \& Organization Management, 37, 137-170. doi:10.1177/1059601111434202

Wright S, Bowkett J, \& Bray K (1995). The communication gap in the ICU--a possible solution. Nursing in Critical Care, 1, 241-244.

Writing Group for the CHECKLIST-ICU Investigators and the Brazilian Research in Intensive Care Network (BRICNet) (2016). Effect of a quality improvement intervention with daily round checklists, goal setting, and clinician prompting on mortality of critically ill patients: A randomized clinical trial. JAMA, 315, 1480-1490. doi:10.1001/jama.2016.3463 [PubMed: 27115264]

Xie A, \& Carayon P (2015). A systematic review of human factors and ergonomics (HFE)-based healthcare system redesign for quality of care and patient safety. Ergonomics, 58, 33-49. doi: 10.1080/00140139.2014.959070 [PubMed: 25323570]

Yoo EJ, Edwards JD, Dean ML, \& Dudley RA (2014). Multidisciplinary care and intensivist staffing: Results from a statewide survey and association with mortality. Journal of Intensive Care Medicine, 31, 325-332. doi:10.1177/0885066614534605 [PubMed: 24825859] 